\title{
Monocytic myeloid-derived suppressor cells as a potent suppressor of tumor immunity in non-small cell lung cancer
}

\author{
KATARZYNA POGODA ${ }^{1-3}$, MARIA PYSZNIAK ${ }^{1-3}$, PAWEŁ RYBOJAD ${ }^{4}$ and JACEK TABARKIEWICZ ${ }^{1,2}$ \\ ${ }^{1}$ Centre for Innovative Research in Medical and Natural Sciences, Faculty of Medicine, University of Rzeszow, \\ 35-310 Rzeszow; ${ }^{2}$ Department of Immunology, Faculty of Medicine, University of Rzeszow, 35-959 Rzeszow; \\ ${ }^{3}$ Postgraduate School of Molecular Medicine, Medical University of Warsaw, 02-091 Warsaw; \\ ${ }^{4}$ Department of Thoracic Surgery, Medical University of Lublin, 20-097 Lublin, Poland
}

Received May 10, 2016; Accepted August 1, 2016

DOI: $10.3892 / \mathrm{ol} .2016 .5273$

\begin{abstract}
Immunotherapy is a promising therapeutic option for patients with non-small cell lung cancer (NSCLC) who do not qualify for surgery. In patients with advanced NSCLC, systemic immune suppression is frequently observed, therefore, researchers are investigating the tumor microenvironment for less invasive and more effective methods of treating lung cancer. Monocytic myeloid-derived suppressor cells (Mo-MDSCs) are potent suppressors of tumor immunity; therefore, this population may significantly impede the application of immunotherapy to treat cancer. The present study evaluated the distribution of Mo-MDSCs and monocytes/macrophages in the peripheral blood, lymph nodes and tumor tissue of patients with NSCLC. Furthermore, the profiles of cytokines produced by these cell populations, including interleukin (IL)-1 $\beta$, IL-12/23p40, IL-10, transforming growth factor- $\beta$ (TGF- $\beta$ ) and tumor necrosis factor (TNF), were compared. The cell populations and the expression of cytokines were assessed by flow cytometry after $4 \mathrm{~h}$ in culture with mitogens and Brefeldin A. Mo-MDSCs were more numerous than monocytes/macrophages in all tissues and their prevalence was highest in the peripheral blood; they expressed higher levels of TGF- $\beta$ than monocytes/macrophages in all
\end{abstract}

Correspondence to: Ms. Katarzyna Pogoda, Centre for Innovative Research in Medical and Natural Sciences, Faculty of Medicine, University of Rzeszow, Warzywna 1a, 35-310 Rzeszow, Poland E-mail: katarzyna.pogoda@o2.pl

Abbreviations: IL-1 $\beta$, interleukin-1 $\beta$; IL-10, interleukin-10; mAbs, monoclonal antibodies; MDSCs, myeloid-derived suppressor cells; Mo-MDSCs, monocytic myeloid-derived suppressor cells; Treg, regulatory T-cell; NHL, non-Hodgkin lymphoma; NSCLC, non-small cell lung cancer; PBMCs, peripheral blood mononuclear cells; TGF- $\beta$, transforming growth factor- $\beta$; TNF, tumor necrosis factor

Key words: non-small cell lung cancer, monocytic-myeloid derived suppressor cells, monocytes/macrophages, flow cytometry, cytokines, IL-1 $\beta$, IL-10, IL-12/23p40, TNF, TGF- $\beta$ tissues and expression of TGF- $\beta$ produced by Mo-MDSCs was higher in the blood than in lymph nodes and tumor tissues. A higher percentage of monocytes/macrophages was observed in lymph nodes and tumor tissues than in blood. CD14 ${ }^{+} \mathrm{HLA}_{-}-\mathrm{DR}^{+}$cells also produced more IL-10 in lymph nodes than Mo-MDSCs and more IL-1 $\beta$ and TNF in all tissues. A higher prevalence of cluster of differentiation $14^{+}$ human leukocyte antigen-D related ${ }^{+}$cells secreting IL-1 $\beta$, TNF and IL-12/23p40 was observed in peripheral blood. Thus, the results of the current study support the statement that Mo-MDSCs and monocytes/macrophages participate in NSCLC induced immunosuppression, and is consistent with previous research into associations between the TGF- $\beta$ signaling pathway and tumor cell invasion, motility and metastasis. The study also demonstrated that Mo-MDSCs promote tumor growth through their immunosuppressive activity. In addition, the profile of cytokines expressed by monocytes/macrophages suggests that this cell population may be associated with metastasis formation and angiogenesis promotion in patients with NSCLC.

\section{Introduction}

Lung cancer is one of the most prevalent types of cancer among women and men worldwide (1). Non-small cell lung cancer (NSCLC) accounts for $>85 \%$ of all cases of lung cancer. NSCLC is primarily treated by surgery (2), however, patients diagnosed in the advanced stages of the disease often may not qualify for surgery. In such cases, immunotherapy may be a promising therapeutic strategy. Although, systemic immune suppression is frequently observed in patients with advanced NSCLC (3). Therefore, researchers are investigating the tumor microenvironment with the aim of making lung cancer treatment less invasive and more effective.

A number of studies have demonstrated that myeloid-origin cells are potent suppressors of tumor immunity, therefore this cell population may significantly impede the application of immunotherapy in cancer (4-7). Researchers have endeavored to determine the best possible phenotype of myeloid-derived suppressor cells (MDSCs) $(8,9)$. Identification of characteristic surface markers of this population is complicated, 
particularly due to its high plasticity and effect on the tumor microenvironment (9). With regards to murine models, the most universal is a combination of Gr-1 protein isoforms and cluster of differentiation (CD) $11 \mathrm{~b}(10,11)$. However, the Gr-1 molecule is not expressed in human MDSCs (12), and researchers must therefore search for a different characteristic surface marker.

Human MDSCs are divided into granulocytic and monocytic (Mo) populations (13). The first group of cells has been described as $\mathrm{CD} 14^{-} \mathrm{CD} 11 \mathrm{~b}^{+} \mathrm{CD} 33^{+} \mathrm{CD} 15^{+}$and the second group as $\mathrm{CD} 14^{+}$human leukocyte antigen-D related (HLA-DR)-/low (13). The absence or low expression of HLA-DR is characteristic in Mo-MDSCs, thus the CD14 ${ }^{+}$HLA-DR $/$low phenotype has been used in studies concerning the identification of Mo-MDSCs in NSCLC $(5,14)$.

Several immunosuppressive functions of MDSCs have been suggested, including the inhibition of dendritic cell differentiation and natural killer cell cytotoxicity, inhibition of T-cell $\left(\mathrm{CD}^{+}\right.$and $\left.\mathrm{CD}^{+}\right)$activation, B-cell impairment and promotion of regulatory T-cell (Treg) expansion. Furthermore, MDSCs may be involved in the promotion of metastasis and angiogenesis $(15,16)$. The presence of MDSCs was confirmed in several forms of cancer, including prostate cancer, glioblastoma, hepatocellular carcinoma, melanoma, squamous cell carcinoma of the head and neck, and NSCLC $(5,12,17,18)$. However, the phenotype and function of MDSCs in NSCLC requires further clarification.

The present study evaluated the distribution of Mo-MDSCs (CD14+HLA-DR-/low) and monocytes/macrophages $\left(\mathrm{CD}^{+} 4^{+} \mathrm{HLA}-\mathrm{DR}^{+}\right)$in peripheral blood, lymph nodes and tumor tissue of patients with NSCLC. Furthermore, the profiles of cytokines produced by Mo-MDSCs and monocytes/macrophages were compared in order to investigate their role in NSCLC. The present study focused on interleukin (IL)-1 $\beta$, IL-10, IL-12/23p40, tumor necrosis factor (TNF) and transforming growth factor- $\beta$ (TGF- $\beta$ ) as they are frequently present in numerous solid tumors and serve important roles in tumor progression. The data obtained during the study identified statistically significant differences between the expression of Mo-MDSCs and monocytes/macrophages in several tissues and the profiles of cytokines secreted by these cell populations.

\section{Materials and methods}

Blood, lymph node and primary tumor samples. Samples were taken from 12 patients with NSCLC, who underwent surgical procedures in the Department of Thoracic Surgery, Independent Public Teaching Hospital No. 4, Medical University of Lublin (Lublin, Poland). Whole blood samples and fragments of lymph nodes and tumor tissue were collected from October 2013 to September 2014. The mean age of patients enrolled in the study was $62.25 \pm 8.44$ years (range, $48-77$ years). Of these 12 patients with histologically confirmed cancer (according to the 7th edition of the Lung Cancer Tumor-Node-Metastasis classification and staging system) (19), 4 were classified as stage IIIA, 5 as stage IIA, 1 as stage IIB and 2 as stage IA or IB (Table I). The number of patients studied was small due to the very strict inclusion criteria, which was as follows: Patients were not allowed to have taken any medication with an immunomodulatory effect in the last month, they were not allowed to have a previous medical history of other autoimmune diseases or cancer, and they must not have presented with any signs of inflammation in the last month. All patients underwent thoracotomy performed by the same surgical team. Blood was drawn immediately prior to surgery, while lymph node and tumor samples were obtained during the surgery. From the whole blood samples, peripheral blood mononuclear cells (PBMCs) were isolated by density gradient centrifugation $\left(700 \times \mathrm{g}\right.$ ) for $20 \mathrm{~min}$ at $20^{\circ} \mathrm{C}$ using Gradisol L (Aqua-Med, Łódź, Poland). Lymph node and tumor samples were cut into small pieces and subsequently homogenized using a gentleMACS ${ }^{\mathrm{TM}}$ Dissociator (Miltenyi Biotec $\mathrm{GmbH}$, Bergisch Gladbach, Germany). Suspensions were subsequently passed through $70 \mu \mathrm{m}$ mesh strainers and separated cells were washed twice with phosphate-buffered saline (PAA Laboratories; GE Healthcare Life Sciences, Chalfont, UK). The study was approved by the ethics committee of the Medical University of Lublin, and written informed consent was obtained from all patients enrolled in the study.

Cell culture. PBMCs, lymph node and tumor cells were cultured in AIM-V ${ }^{\circledR}$ medium (Gibco; Thermo Fisher Scientific, Inc., Waltham, MA, USA) for $4 \mathrm{~h}$ with the addition of ionomycin, phorbol myristate acetate, lipopolysaccharide and Brefeldin A (Sigma-Aldrich; Merck Millipore, Darmstadt, Germany). Cells were cultured at $37^{\circ} \mathrm{C}$ in a humidified atmosphere $(95 \%)$ with $5 \% \mathrm{CO}_{2}$.

Flow cytometry. The following fluorochrome-labeled monoclonal antibodies (mAbs), which were purchased from BioLegend, Inc. (San Diego, CA, USA), were used: Phycoerythrin cyanine 7 anti-human CD14 (200 $\mu \mathrm{g} / \mathrm{ml}$; \#325618) and Pacific Blue anti-human HLA-DR (200 $\mu \mathrm{g} / \mathrm{ml}$; \#307633). In addition, the following monoclonal antibodies conjugated with Alexa Fluor 647 were used: Anti-human IL-1 $\beta$ (50 $\mu \mathrm{g} / \mathrm{ml}$; \#508208), anti-human IL-10 (50 $\mu \mathrm{g} / \mathrm{ml}$; \#501412), anti-human IL-12/23p40 (25 $\mu \mathrm{g} / \mathrm{ml}$; \#501818), anti-human TNF $(25 \mu \mathrm{g} / \mathrm{ml}$; \#502916) and anti-human latency-associated peptide (TGF- $\beta 1)(400 \mu \mathrm{g} / \mathrm{ml}$; \#341801). Non-specific Fc receptor-mediated antibody binding was blocked with the use of Human TruStain FcX ${ }^{\mathrm{TM}}$ (BioLegend, Inc.). Subsequently, PBMCs, lymph node and tumor cells were incubated with anti-CD14 and anti-HLA-DR mAbs in the dark for $20 \mathrm{~min}$ at room temperature. Following surface staining, cells were fixed with Fixation Buffer (BioLegend, Inc.) and permeabilized with Intracellular Staining Permeabilization Wash Buffer (BioLegend, Inc.). Intracellular cytokine staining was then performed using anti-IL-1 $\beta$, anti-IL-10, anti-IL-12/23p40, anti-TNF or anti-TGF- $\beta 1 \mathrm{mAbs}$. Data acquisition and analysis were performed on a BD FACSCanto $^{\mathrm{TM}}$ II Flow Cytometer (BD Biosciences, Franklin Lakes, NJ, USA). Representative examples of cytometric analysis are presented in Fig. 1. Results were generated using FACSDiva v8.0.1 software (BD Biosciences).

Statistical analysis. Statistical analyses were performed using STATISTICA v10.0 software (StatSoft, Inc., Tulsa, OK, USA). The data collected in the current study did not meet with assumptions of Gaussian distribution, therefore non-parametric 
Table I. Clinicopathological features of patients with non-small cell lung cancer.

\begin{tabular}{|c|c|c|c|c|c|c|}
\hline Patient & $\begin{array}{l}\text { Age, } \\
\text { years }\end{array}$ & Gender & Smoker & Type of surgery & Stage & Histopathology \\
\hline 1 & 77 & Male & Yes & Thoracotomy, lymphadenectomy & IIIA & Carcinoma planoepitheliale \\
\hline 2 & 55 & Female & Yes & Thoracotomy, lobectomy and lymphadenectomy & IIA & Adenocarcinoma \\
\hline 3 & 60 & Male & Yes & Lobectomy and lymphadenectomy & IIIA & Adenocarcinoma \\
\hline 4 & 64 & Male & Yes & Thoracotomy and lobectomy & IIA & Adenocarcinoma \\
\hline 5 & 73 & Male & Yes & Thoracotomy and lobectomy & IIIA & Carcinoma planoepitheliale \\
\hline 6 & 71 & Male & Yes & Thoracotomy, lobectomy and lymphadenectomy & IB & Carcinoma planoepitheliale \\
\hline 7 & 64 & Male & Yes & $\begin{array}{l}\text { Thoracotomy, pulmonectomy and } \\
\text { lymphadenectomy }\end{array}$ & IIB & Carcinoma planoepitheliale \\
\hline 8 & 48 & Male & Yes & Pulmonectomy & IIIA & Carcinoma planoepitheliale \\
\hline 9 & 62 & Male & Yes & Lobectomy & IA & Adenocarcinoma \\
\hline 10 & 63 & Male & Yes & Lobectomy & IIA & Carcinoma macrocellulare \\
\hline 11 & 49 & Male & Yes & Lobectomy & IIA & Carcinoma macrocellulare \\
\hline 12 & 61 & Female & Yes & $\begin{array}{l}\text { Thoracotomy, lobectomy and } \\
\text { lymphadenectomylymphadenectomy }\end{array}$ & IIA & Adenocarcinoma \\
\hline
\end{tabular}

tests were used to analyze the results. Differences between particular tissues were determined by the analysis of variance (ANOVA) Friedman test and Kendall's W-normalization of the statistic of the Friedman test followed by post-hoc tests. Comparisons between two variables were prepared with the use of Wilcoxon signed-rank test. Results were presented as the median and interquartile range, and $\mathrm{P}<0.05$ was considered to indicate a statistically significant result.

\section{Results}

Distribution of Mo-MDSCs and monocytes/macrophages in the peripheral blood, lymph nodes and tumor tissues. Using flow cytometry analysis, two cell populations were detected with the following immunophenotypes: CD14+HLA-DR $/$ low and $\mathrm{CD} 14^{+} \mathrm{HLA}-\mathrm{DR}{ }^{+}$. While $\mathrm{CD} 14^{+} \mathrm{HLA}-\mathrm{DR}^{+}$is characteristic for populations of monocytes/macrophages, in the current study, low expression of major histocompatibility complex class II among $\mathrm{CD} 14^{+}$cells was observed on the surface of the Mo-MDSCs (CD14+HLA-DR-/low $)$. The population of Mo-MDSCs was significantly higher than the population of monocytes/macrophages in all tissues (blood, $\mathrm{P}=0.005$; lymph nodes, $\mathrm{P}=0.025$; tumor, $\mathrm{P}=0.017$; Fig. 2). The highest percentage of Mo-MDSCs was identified in the peripheral blood and the lowest was in the lymph nodes. According to post-hoc tests, which followed the Friedman ANOVA test, the level of Mo-MDSCs was significantly higher in the blood than the lymph nodes $(\mathrm{P}=0.001)$ and tumor tissue $(\mathrm{P}=0.001)$. By contrast, the highest percentage of monocytes/macrophages was detected in lymph nodes and the lowest in peripheral blood. The prevalence of monocytes/macrophages in lymph nodes and tumor tissue in comparison with peripheral blood was also statistically significant ( $\mathrm{P}=0.001$; Fig. 2).

Comparison of profiles of cytokines produced by Mo-MDSCs and monocytes/macrophages in the peripheral blood, lymph nodes and tumor tissues. The present study demonstrated that each population, CD14+HLA-DR-/low and CD14+HLA-DR+, produced IL-1 $\beta$, IL-10, IL-12/23p40, TNF and TGF- $\beta$ in all examined tissues of patients with NSCLC. However, significant differences between particular tissues were identified in the proportion of Mo-MDSCs and monocytes/macrophages producing these cytokines (Table II).

The percentage of Mo-MDSCs producing TGF- $\beta$ was significantly higher in the blood than in other tissues $(\mathrm{P}=0.001)$. Peripheral blood was characterized by a higher proportion of monocytes/macrophages producing TGF- $\beta$ $(\mathrm{P}=0.002)$ than lymph nodes, and a higher proportion of monocytes/macrophages producing IL-1 $\beta$ than tumor tissue $(\mathrm{P}=0.002)$ (Table II). The post-hoc test did not identify a significant difference between blood and lymph nodes in the proportion of monocytes/macrophages producing TNF. However, the mean fluorescence intensity (MFI) value for this population producing TNF was significantly higher in blood ( $\mathrm{P}=0.008$; data not shown). No statistically significant differences were observed among the level of Mo-MDSCs and monocytes/macrophages or in the expression of cytokines in the post-hoc tests between lymph nodes and tumor tissue.

Analysis ofdifferences between the levels of cytokines produced by the Mo-MDSCs and monocytes/macrophages in the peripheral blood, lymph nodes and tumor tissues. In order to analyze the differences between levels of cytokines produced by the Mo-MDSCs and monocytes/macrophages in several tissues, the Wilcoxon signed-rank test was used. A significantly lower proportion of monocytes/macrophages produced TGF- $\beta$ in the blood $(\mathrm{P}=0.028)$, lymph nodes $(\mathrm{P}=0.015)$ and tumor tissue $(\mathrm{P}=0.006)$ compared with the Mo-MDSCs (Fig. 3). Furthermore, in the lymph nodes, a significantly higher proportion of IL-10 was produced by monocytes/macrophages than the Mo-MDSCs ( $\mathrm{P}=0.003$; Fig. 4). 
Table II. Percentage of cells expressing cytokines in the peripheral blood, lymph nodes and tumor tissue.

\begin{tabular}{|c|c|c|c|c|c|c|}
\hline \multirow[b]{2}{*}{ Cytokines, $\%$} & \multicolumn{2}{|c|}{ Peripheral blood } & \multicolumn{2}{|c|}{ Lymph nodes } & \multicolumn{2}{|c|}{ Tumor tissue } \\
\hline & $\begin{array}{l}\text { Mo-MDSCs, } \\
\text { median } \\
\text { (IQR) }\end{array}$ & $\begin{array}{c}\text { Monocytes/ } \\
\text { macrophages, } \\
\text { median (IQR) }\end{array}$ & $\begin{array}{l}\text { Mo-MDSCs, } \\
\text { median } \\
\text { (IQR) }\end{array}$ & $\begin{array}{l}\text { Monocytes/ } \\
\text { macrophages, } \\
\text { median (IQR) }\end{array}$ & $\begin{array}{l}\text { Mo-MDSCs, } \\
\text { median } \\
\text { (IQR) }\end{array}$ & $\begin{array}{l}\text { Monocytes/ } \\
\text { macrophages, } \\
\text { median (IQR) }\end{array}$ \\
\hline IL-1 $\beta$ & $5.30(18.93)$ & $36.75(37.00)^{\mathrm{d}}$ & $0.90(0.85)$ & $1.70(1.45)$ & $1.10(2.05)$ & $5.50(5.50)^{\mathrm{d}}$ \\
\hline IL-10 & $0.20(0.50)$ & $0.15(1.28)$ & $0.65(1.00)$ & $1.90(2.65)$ & $0.20(0.45)$ & $0.20(0.35)$ \\
\hline IL-12/23p40 & $0.00(0.50)$ & $0.00(0.70)$ & $1.00(1.00)$ & $1.30(2.20)$ & $1.00(0.75)$ & $1.50(2.50)$ \\
\hline TNF & $12.50(17.80)$ & $34.00(28.00)$ & $18.30(31.53)$ & $35.75(32.55)$ & $2.90(11.85)$ & $6.40(13.25)$ \\
\hline TGF- $\beta$ & $38.55(28.48)^{\mathrm{a}, \mathrm{b}}$ & $19.70(0.70)^{\mathrm{c}}$ & $2.15(3.95)^{\mathrm{a}}$ & $0.70(0.90)^{\mathrm{c}}$ & $5.80(23.40)^{\mathrm{b}}$ & $3.80(9.50)$ \\
\hline
\end{tabular}

${ }^{\text {aD }}$ ifference between median of Mo-MDSCs producing TGF- $\beta$ in peripheral blood and lymph nodes $(\mathrm{P}=0.001)$; ${ }^{\mathrm{b}}$ difference between median

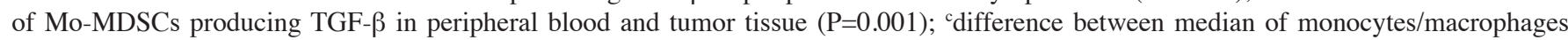
producing TGF- $\beta$ in peripheral blood and lymph nodes $(\mathrm{P}=0.002)$; ${ }^{d}$ difference between median of monocytes/macrophages producing IL-1 $\beta$ in peripheral blood and tumor tissue $(\mathrm{P}=0.002)$. Mo-MDSCs, monocytic myeloid-derived suppressor cells; IQR, interquartile range; IL, interleukin; TNF, tumor necrosis factor; TGF, transforming growth factor.

A greater level of IL-1 $\beta$ was produced by the CD14 ${ }^{+}$HLA-DR ${ }^{+}$population in comparison with Mo-MDSCs in all tissues, but the only significant result was observed in the blood ( $\mathrm{P}=0.004$; Fig. 5A). Also the MFI value for monocytes/macrophages producing IL-1 $\beta$ in peripheral blood was significantly higher than the MFI value for Mo-MDSCs producing IL-1 $\beta(\mathrm{P}=0.005$; Fig. $5 \mathrm{~B})$. Differences in expression of IL-1 $\beta$ in the tumor and lymph nodes were not significant (lymph nodes $\mathrm{P}=0.074$; tumor $\mathrm{P}=0.062$; Fig. $5 \mathrm{C}$ and $\mathrm{D}$, respectively). Statistical analysis demonstrated a higher percentage of monocytes/macrophages producing TNF in peripheral blood and tumor tissue (Fig. 6A and D, respectively) and a higher MFI value for the mentioned population in the blood and lymph nodes (Fig. 6B and C, respectively). However, a significant difference was only observed between MFI values measured for monocytes/macrophages and Mo-MDSCs producing TNF in the blood ( $\mathrm{P}=0.008$; Fig. 6B). Data analysis indicated that in lymph nodes and tumor tissue, monocytes/macrophages produced a higher proportion of IL-12/23p40 than Mo-MDSCs $(\mathrm{P}=0.047$ and $\mathrm{P}=0.041$, respectively; Fig. 7).

\section{Discussion}

Lung cancer is the most prevalent cause of cancer-associated mortality in the world (20). The most common subtype is NSCLC, which is characterized by poor prognosis and high mortality, particularly in cases that are unsuitable for surgery (1). A number of treatment options are currently available to treat patients with cancer, however, therapy is often ineffective. A growing body of evidence suggests that cancer initiation and progression depends on the ability of cancer cells to evade host immunosurveillance $(21,22)$. Cancer cells regulate and modify immune responses and therefore contribute to an imbalance in type 1 T-helper (Th)/Th2 responses (21) and the enhancement of immunosuppressive cells, including Treg cells (23), tumor associated macrophages (24), type 2 natural killer T-cells (25) and myeloid-derived suppressor cells (26). Therapeutic strategies targeting these cell populations are emerging (27-31) and may benefit patients in the future. However, prior to the therapeutic exploitation of these cell populations, it is important to evaluate their role and interactions in the tumor microenvironment. The current study therefore aimed to investigate the distribution of Mo-MDSCs and monocytes/macrophages in NSCLC tissues, and additionally assess and compare the profiles of cytokines produced by these cell populations. To the best of our knowledge, this is the first study to examine the distribution of Mo-MDSCs and monocytes/macrophages simultaneously in the peripheral blood, tumor tissues and lymph nodes of patients with NSCLC.

In the present study, Mo-MDSCs and monocytes/macrophages were detected in all examined tissues. Mo-MDSCs were more abundant than monocytes/macrophages throughout all tissues, particularly in peripheral blood. Vetsika et al (15) previously identified an inverse correlation between CD14 ${ }^{+}$HLA-DR $/$low and CD14 ${ }^{+} \mathrm{HLA}^{-} \mathrm{DR}^{+}$cells in the peripheral blood of patients with NSCLC. This suggests that $\mathrm{CD}^{+} 4^{+} \mathrm{HLA}-\mathrm{DR}^{+}$cells are effector cells, which are responsible for activating T lymphocytes (15). However, Feng et al (32) demonstrated that in patients with NSCLC, the mixture of CD14 ${ }^{+}$HLA-DR $/$low cells and CD14 ${ }^{+} \mathrm{HLA}^{-} \mathrm{DR}^{+}$cells resulted in a more powerful suppressive activity on T-cells than the $\mathrm{CD}^{+} 4^{+} \mathrm{HLA}-\mathrm{DR}-\mathrm{low}$ population alone (32). The immunosuppressive activity of Mo-MDSCs and CD14 ${ }^{+} \mathrm{HLA}-\mathrm{DR}^{+}$cells was confirmed in multiple myeloma (33). By contrast, Schilling et al (14) demonstrated that CD14+HLA-DR ${ }^{+}$cells did not exhibit suppressive properties in melanoma.

In the present study, it was observed that monocytes/macrophages were significantly more prevalent in the lymph nodes and tumor tissues than in blood. This result supports the statement that Mo-MDSCs may differentiate into monocytes/macrophages in the tumor microenvironment (34). This process may be regulated by hypoxia inducible factor $1 \alpha$, which is induced in the tumor microenvironment by hypoxia $(34,35)$. 
A

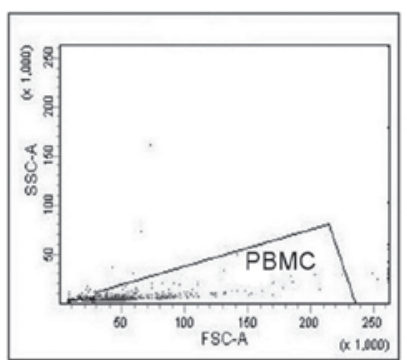

$\mathrm{C}$
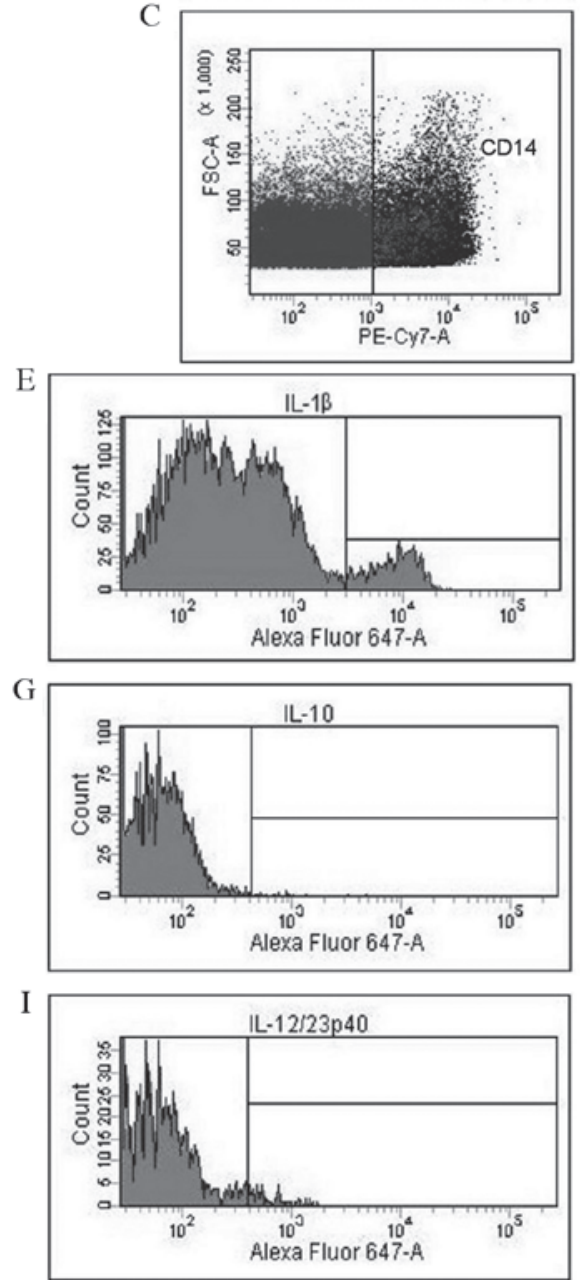

$\mathrm{K}$
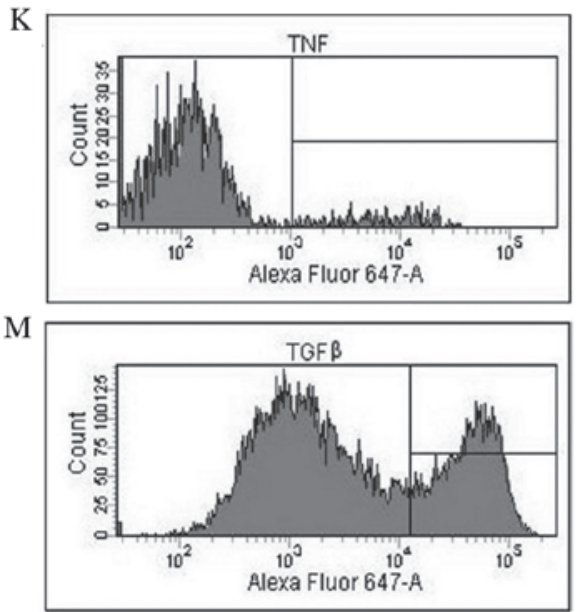

$\mathrm{B}$

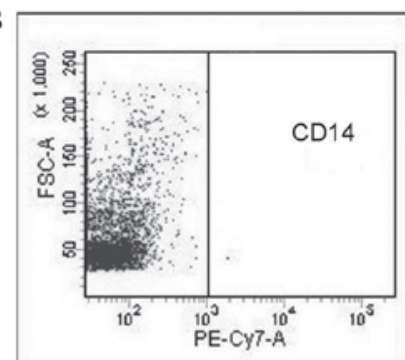

D
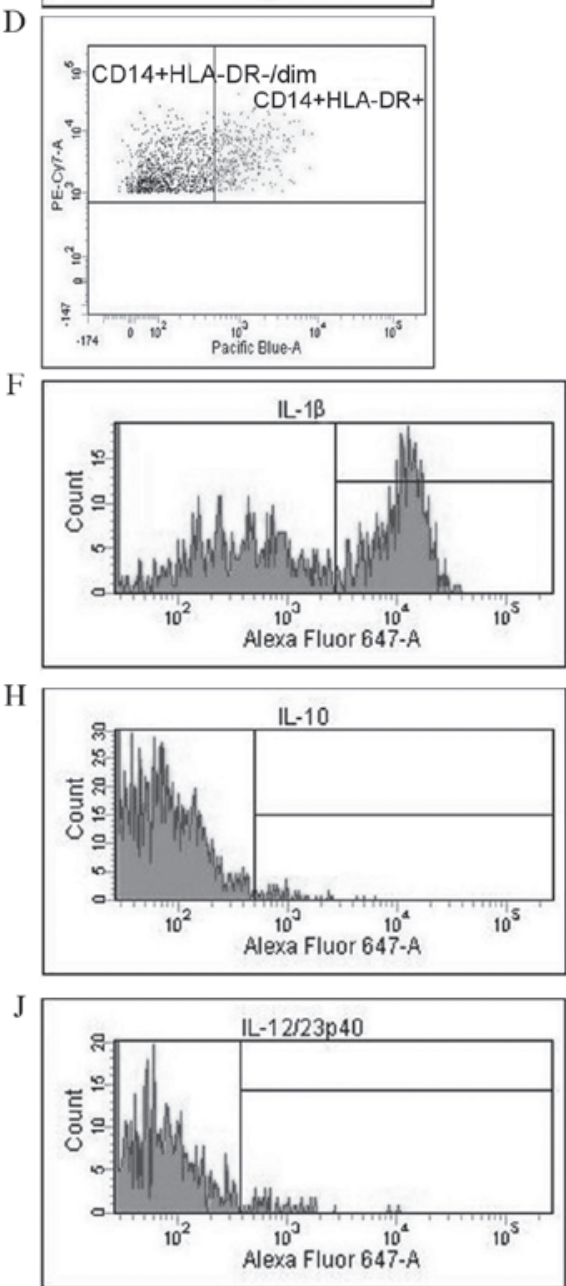

$\mathrm{L}$
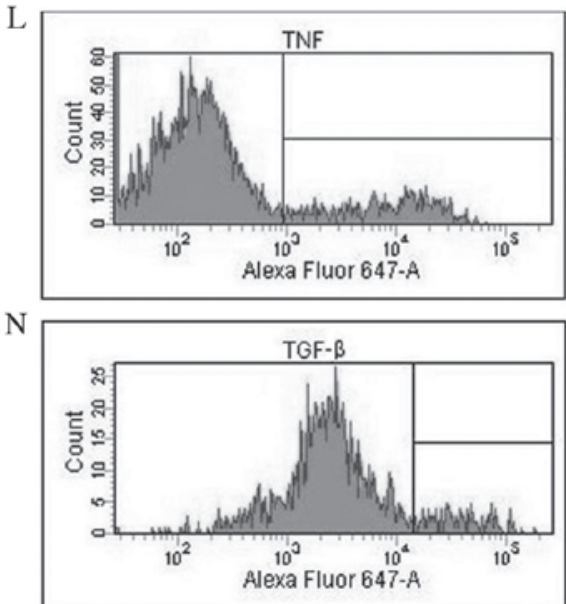

Figure 1. Analysis of cytokine profiles and evaluation of the expression of Mo-MDSCs and monocytes/macrophages in patients with non-small cell lung cancer with the use of flow cytometry. (A) Gate for PBMCs, (B) CD14 negative control, (C) identification of CD14 ${ }^{+}$cells and (D) differentiation of CD14 $4^{+}{ }^{-}{ }^{-}{ }^{-} /$low cells (Mo-MDSCs) and CD14 ${ }^{+} \mathrm{HLA}^{-} \mathrm{DR}^{+}$cells (monocytes/macrophages). Evaluation of percentage of Mo-MDSCs producing (E) IL-1 $\beta$, (G) IL-10, (I) IL-12/23p40, (K) TNF and (M) TGF- $\beta$. Assessment of percentage of monocytes/macrophages producing (F) IL-1 $\beta$, (H) IL-10, (J) IL-12/23p40, (L) TNF and (N) TGF- $\beta$. Mo-MDSCs, monocytic myeloid-derived suppressor cells; PBMC, peripheral blood mononuclear cells; CD, cluster of differentiation; IL, interleukin; TNF, tumor necrosis factor; TGF- $\beta$, transforming growth factor- $\beta$. 

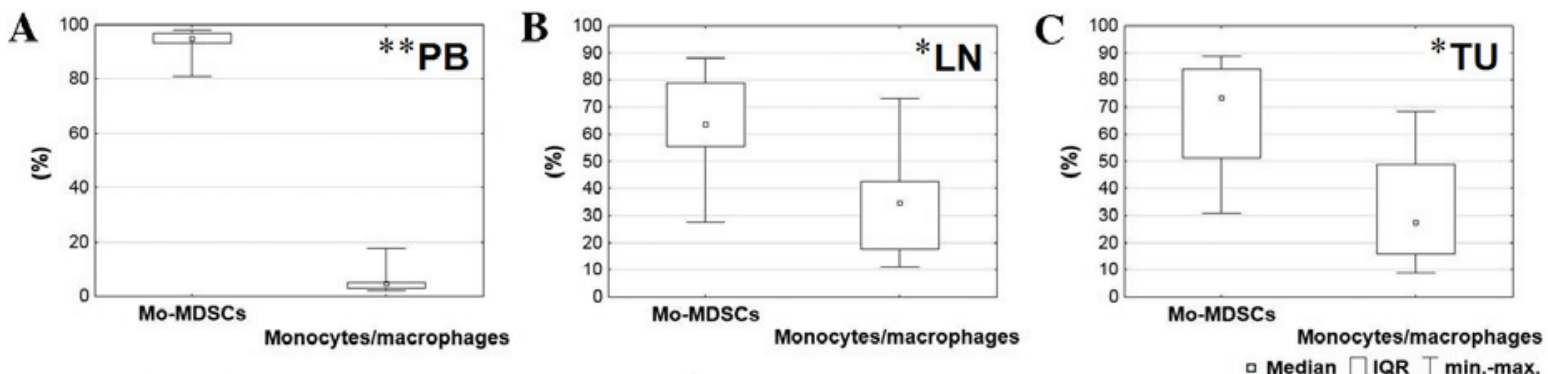

Figure 2. Comparison between percentages of Mo-MDSCs and monocytes/macrophages observed in the (A) peripheral blood, (B) lymph nodes and (C) tumor tissue. " $\mathrm{P}<0.05$ and ${ }^{* *} \mathrm{P}<0.01$. LN, lymph nodes; Mo-MDSCs, monocytic myeloid-derived suppressor cells; IQR, interquartile range; PB, peripheral blood; TU, tumor tissue.
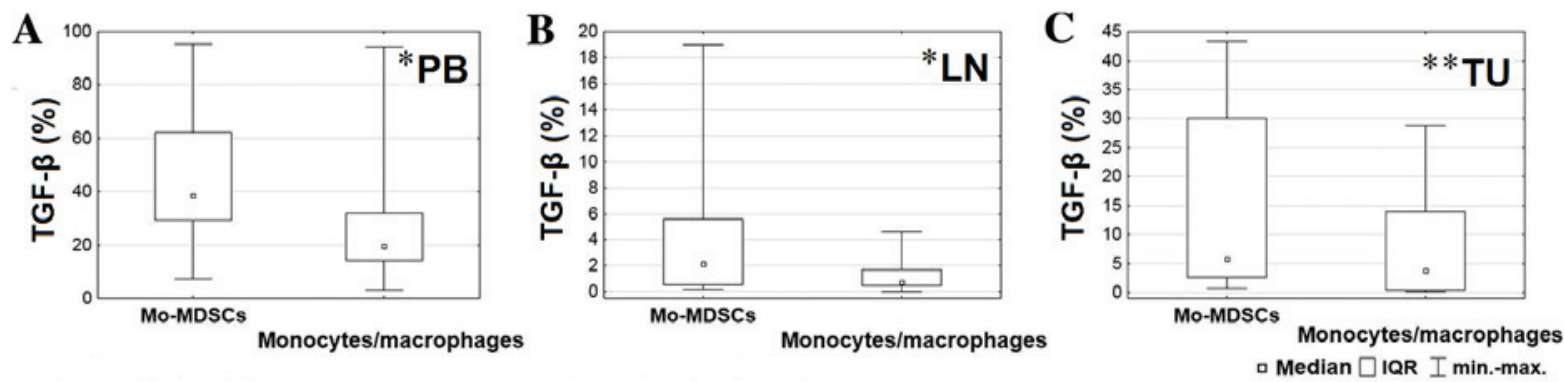

Figure 3. Comparison between percentages of Mo-MDSCs and monocytes/macrophages producing transforming growth factor- $\beta$ in the (A) peripheral blood, (B) lymph nodes and (C) tumor tissue. " $\mathrm{P}<0.05$ and ${ }^{* *} \mathrm{P}<0.01$. LN, lymph nodes; Mo-MDSCs, monocytic myeloid-derived suppressor cells; IQR, interquartile range; $\mathrm{PB}$, peripheral blood; TGF- $\beta$, transforming growth factor- $\beta$; TU, tumor tissue.

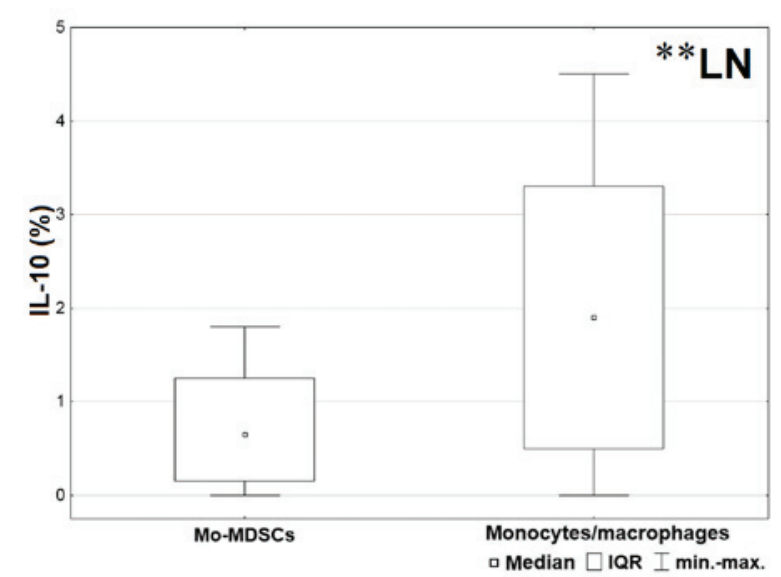

Figure 4. Comparison between percentages of Mo-MDSCs and monocytes/macrophages producing interleukin-10 in the lymph nodes. ${ }^{* *} \mathrm{P}<0.01$. LN, lymph nodes; Mo-MDSCs, monocytic myeloid-derived suppressor cells; IQR, interquartile range; PB, peripheral blood; TU, tumor tissue.

TGF- $\beta$ may stimulate tumor progression in advanced stages of tumorigenesis, and is described as an immunosuppression and angiogenesis promoter in the tumor microenvironment (36). Beury et al (37) and De Keersmaecker et al (33) reported that TGF- $\beta$ is produced by Mo-MDSCs and monocytes/macrophages in the peripheral blood. However, it may be necessary to closely analyze the cross-talk and differences between MDSCs and monocytes/macrophages, and investigate the distribution of TGF- $\beta$ in the tissues of patients with cancer. At the protein level, FACS data obtained during the present study indicated a significantly higher number of Mo-MDSCs producing TGF- $\beta$ in all tissues. However, a previous study by Huang et al (5) did not observe any differences in the transcription of this

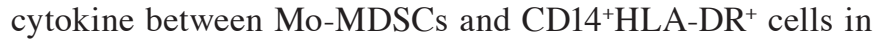
the peripheral blood of patients with NSCLC.

The presence of IL-10 has been confirmed in various types of cancer, including B-cell non-Hodgkin lymphoma (NHL), prostate cancer, melanoma, squamous cell carcinoma of the head and neck, hepatocellular carcinoma, multiple myeloma, glioblastoma and bladder carcinoma $(5,38-40)$. According to Feng et al (32), IL-10 is responsible for upregulating arginase 1 and therefore inhibits T-cell activation in patients with NSCLC. Furthermore, Xiu et al (40) demonstrated that IL-10 is responsible for the development of Mo-MDSCs in B-cell NHL. Elevated production of IL-10 by macrophages associated with the tumor microenvironment correlates with poor prognosis, stage of disease, tumor size and lymph node metastasis $(38,41)$. This correlation was confirmed in patients with NSCLC $(42,43)$. However, there was no clear division of cells into $\mathrm{CD} 14^{+} \mathrm{HLA}-\mathrm{DR}-1$ low and $\mathrm{HLA}-\mathrm{DR}^{+}$populations in the aforementioned studies. The results of the current study demonstrated that Mo-MDSCs and monocytes/macrophages produced IL-10 in all examined tissues of patients with NSCLC. Furthermore, a significantly higher proportion of monocytes/macrophages producing IL-10 than Mo-MDSCs were observed in lymph nodes; this is important as lymph 
A

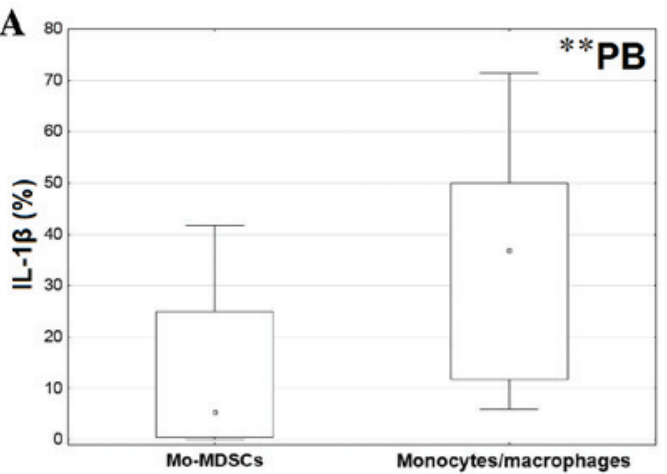

C

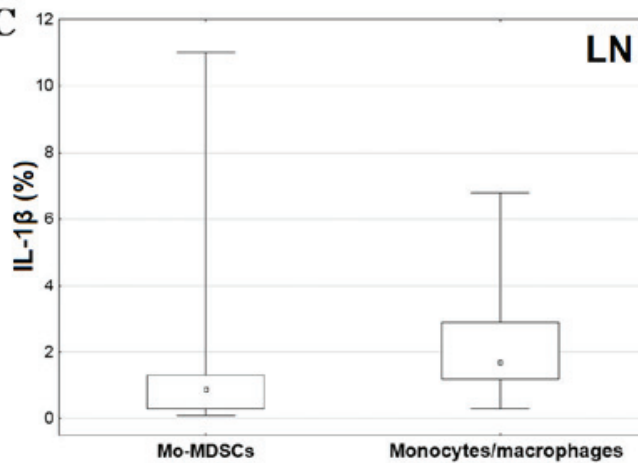

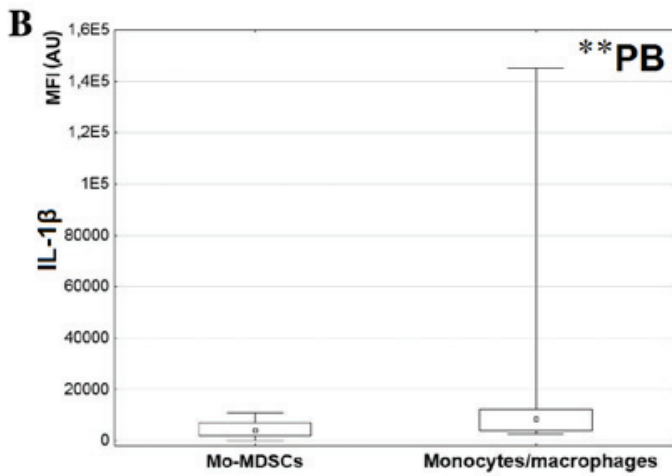

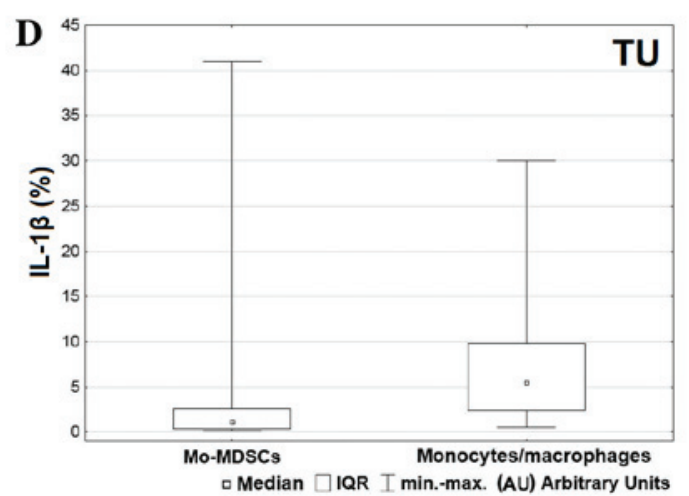

Figure 5. Comparison between percentages of Mo-MDSCs and monocytes/macrophages producing IL-1 $\beta$ in the (A) peripheral blood, (C) lymph nodes and (D) tumor tissue; (B) comparison of MFI value between Mo-MDSCs and monocytes/macrophages producing IL-1 $\beta$ in peripheral blood. ${ }^{* *} \mathrm{P}<0.01$. LN, lymph nodes; Mo-MDSCs, monocytic myeloid-derived suppressor cells; IL, interleukin; MFI, mean fluorescence intensity; IQR, interquartile range; PB, peripheral blood; TU, tumor tissue.

A
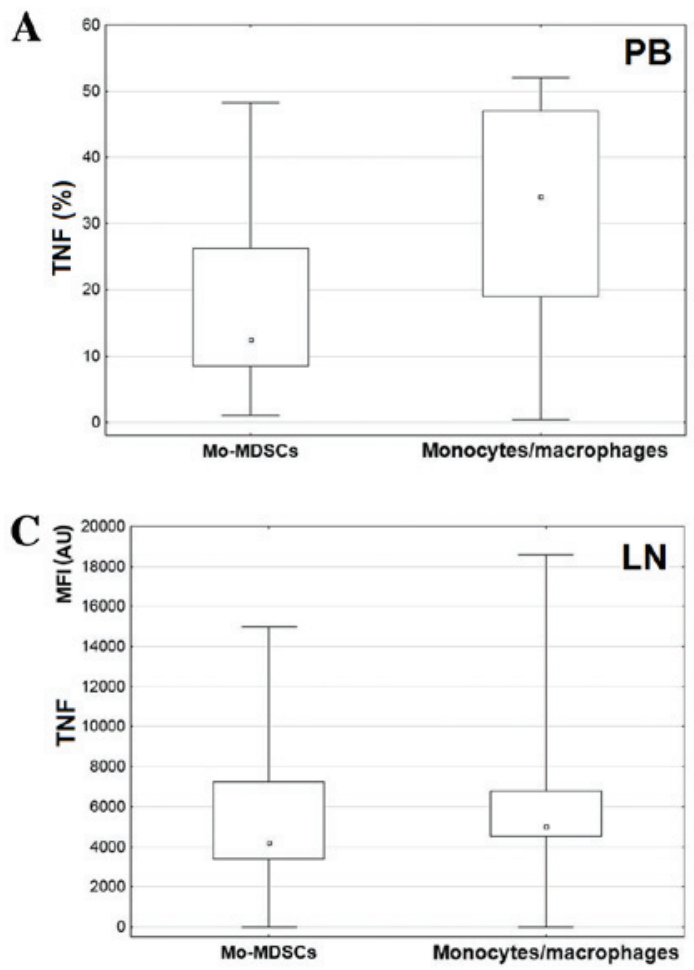

B

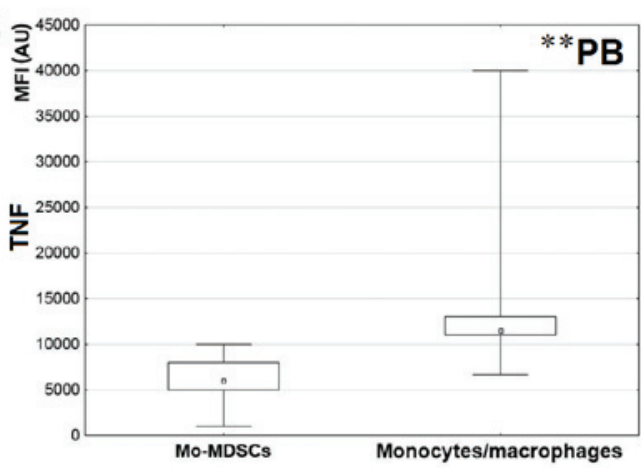

D

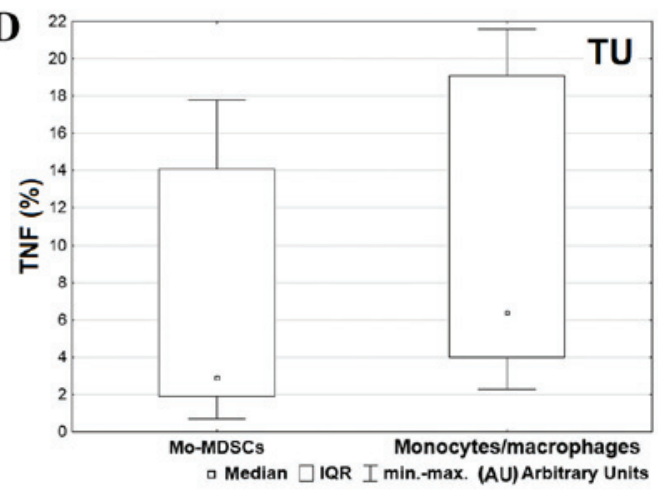

Figure 6. Comparison between percentages of Mo-MDSCs and monocytes/macrophages producing TNF in the (A) peripheral blood and (D) tumor tissue. Comparison of MFI value between Mo-MDSCs and monocytes/macrophages producing TNF in the (B) peripheral blood and (C) lymph nodes. ${ }^{* *} \mathrm{P}<0.01$. LN, lymph nodes; Mo-MDSCs, monocytic myeloid-derived suppressor cells; TNF, tumor necrosis factor; MFI, mean fluorescence intensity; IQR, interquartile range; $\mathrm{PB}$, peripheral blood; $\mathrm{TU}$, tumor tissue. 
A

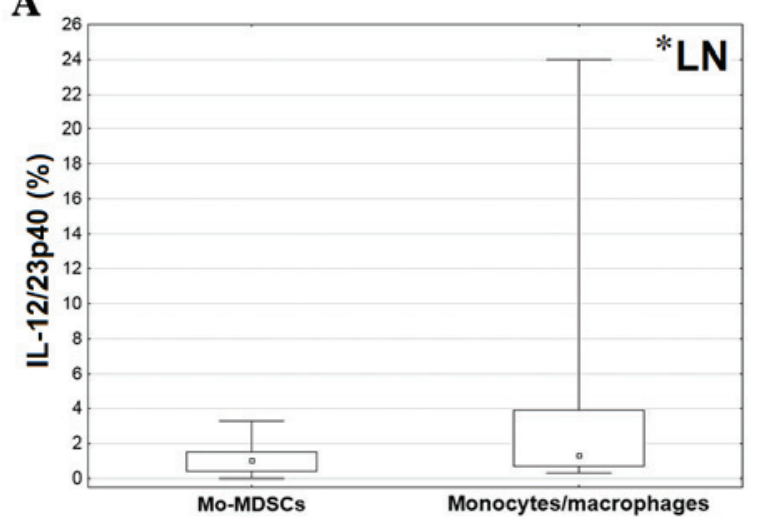

B

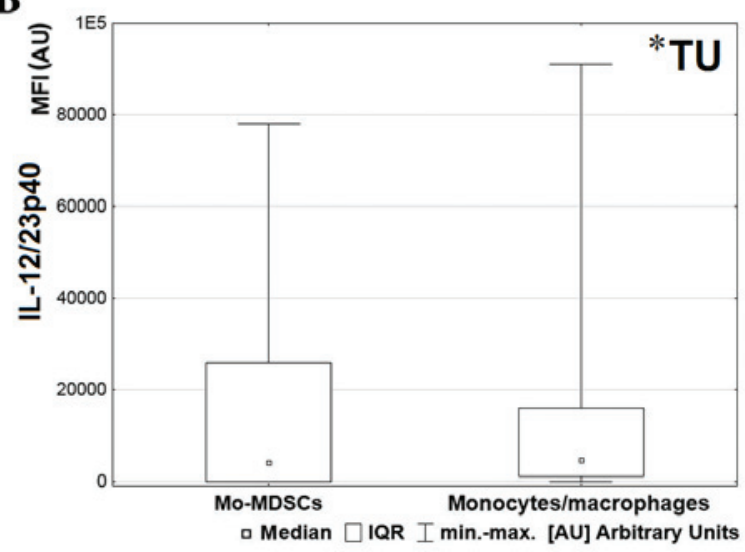

Figure 7. (A) Comparison between percentages of Mo-MDSCs and monocytes/macrophages producing IL-12/23p40 in the lymph nodes and (B) comparison of MFI value between Mo-MDSCs and monocytes/macrophages producing IL-12/23p40 in tumor tissues. "P<0.05. LN, lymph nodes; Mo-MDSCs, monocytic myeloid-derived suppressor cells; IL, interleukin; MFI, mean fluorescence intensity; IQR, interquartile range; PB, peripheral blood; TU, tumor tissue.

nodes are sites of lymphocyte activation and immune response induction.

According to the results of the present study, monocytes/macrophages have the ability to produce higher levels of IL-1 $\beta$, TNF and IL-12/23p40 than Mo-MDSCs. A significantly higher prevalence of $\mathrm{CD} 14^{+} \mathrm{HLA}-\mathrm{DR}^{+}$producing IL- $1 \beta$ and TNF was observed in peripheral blood and a significantly higher prevalence of $\mathrm{CD} 14^{+} \mathrm{HLA}-\mathrm{DR}^{+}$cells producing IL-12/23p40 was observed in lymph nodes and tumor tissue. These observations correspond to the cytokine functions: IL-1 $\beta$ and TNF are responsible for generating MDSCs in the bone marrow (44), and both cytokines have a proangiogenic role in patients with NSCLC (45). The p40 subunit is common for IL-12 and IL-23, therefore, both cytokines had to be considered in the analysis. IL-12 promotes Th 1 immunity and inhibits angiogenesis in the tumor microenvironment, while IL-23 exhibits tumor-promoting effects (46). According to the results of research on cross-talk between MDSCs and macrophages in solid tumors, each population produces IL-12 $(37,47)$. However, in the current in vitro study, IL-23p40 was not produced. By contrast, Baird et al (48) confirmed the presence of IL-23 in the tumor microenvironment and demonstrated that IL-23 induced proliferation in an NSCLC cell line and promoted proliferation in primary NSCLC tumors (48). In the present study, a higher prevalence of IL-12/23p40 positive cells in lymph nodes and in tumor tissue was observed. This may be associated with a more aggressive tumor, when the results of Baird et al (48) are considered.

In conclusion, the present study demonstrated that Mo-MDSCs were more abundant than monocytes/macrophages in all NSCLC tissue examined and the highest percentage of Mo-MDSCs was detected in the blood.In all tissues, Mo-MDSCs produced higher levels of TGF- $\beta$ than $\mathrm{CD} 14^{+} \mathrm{HLA}^{-D R^{+}}$cells. Furthermore, the proportion of Mo-MDSCs producing TGF- $\beta$ was higher in the blood compared with the lymph nodes and tumor tissues. These observations suggest that Mo-MDSCs and monocytes/macrophages participate in NSCLC induced immunosuppression. In addition, higher levels of Mo-MDSCs and TGF- $\beta$ identified in the blood may correspond with results from previous studies regarding associations between the TGF- $\beta$ signaling pathway and tumor cell invasion, motility and metastasis, and also with the notion that Mo-MDSCs promote tumor growth through their immunosuppressive activity. Lymph nodes and tumor tissue were characterized by a higher prevalence of monocytes/macrophages than peripheral blood. This population produced significantly more immunosuppressive IL-10 in lymph nodes than Mo-MDSCs. CD14+HLA-DR ${ }^{+}$ cells secreted more IL-1 $\beta$ and TNF than Mo-MDSCs in all tissues. Furthermore, a higher proportion of monocytes/macrophages producing cytokines was observed in the peripheral blood. This corresponds with the fact that IL-1 $\beta$ and TNF produced by monocytes/macrophages are associated with metastasis formation and angiogenesis promotion. A higher percentage of IL-12/23p40 produced by CD14 ${ }^{+} \mathrm{HLA}_{-}-\mathrm{DR}^{+}$ cells was detected in lymph nodes and in tumor tissue. This molecule may represent IL-23, which promotes proliferation in primary NSCLC tumors. Analyzing the distribution of Mo-MDSCs and monocytes/macrophages in NSCLC tissues and profiling the cytokines they secrete may allow researchers to investigate the role of these populations in the tumor microenvironment and subsequently enable modifications to their functions. In the future, modulating the activities of MDSCs may be a useful additional tool in anticancer therapy.

\section{Acknowledgements}

The present study was performed within the project 'Centre for Innovative Research in Medical and Natural Sciences' initiated by the University of Rzeszów (Rzeszów, Poland), and was co-financed by the Regional Operational Programme for the Podkarpackie Province between 2007 and 2013 (contract number, UDA-RPPK.01.03.00-18-004/12-00).

\section{References}

1. Nur U, Quaresma M, De Stavola B, Peake M and Rachet B: Inequalities in non-small cell lung cancer treatment and mortality. J Epidemiol Community Health 69: 985-992, 2015. 
2. Thomas A, Chen Y, Yu T, Jakopovic M and Giaccone G: Trends and characteristics of young non-small cell lung cancer patients in the United States. Front Oncol 5: 113, 2015.

3. Aerts JG, Lievense LA, Hoogsteden HC and Hegmans JP: Immunotherapy prospects in the treatment of lung cancer and mesothelioma. Transl Lung Cancer Res 3: 34-45, 2014.

4. Ohki S, Shibata M, Gonda K, Machida T, Shimura T, Nakamura I, Ohtake T, Koyama Y, Suzuki S, Ohto H and Takenoshita S: Circulating myeloid-derived suppressor cells are increased and correlate to immune suppression, inflammation and hypoproteinemia in patients with cancer. Oncol Rep 28: 453-458, 2012.

5. Huang A, Zhang B, Wang B, Zhang F, Fan KX and Guo YJ: Increased CD14(+)HLA-DR (-/low) myeloid-derived suppressor cells correlate with extrathoracic metastasis and poor response to chemotherapy in non-small cell lung cancer patients. Cancer Immunol Immunother 62: 1439-1451, 2013.

6. Ostrand-Rosenberg S: Myeloid-derived suppressor cells: More mechanisms for inhibiting antitumor immunity. Cancer Immunol Immunother 59: 1593-1600, 2010

7. Parker KH, Beury DW and Ostrand-Rosenberg S: Myeloid-Derived suppressor cells: Critical cells driving immune suppression in the tumor microenvironment. Adv Cancer Res 128: 95-139, 2015.

8. Marigo I, Dolcetti L, Serafini P, Zanovello P and Bronte V: Tumor-induced tolerance and immune suppression by myeloid derived suppressor cells. Immunol Rev 222: 162-179, 2008.

9. Damuzzo V, Pinton L, Desantis G, Solito S, Marigo I, Bronte V and Mandruzzato S: Complexity and challenges in defining myeloid-derived suppressor cells. Cytometry B Clin Cytom 88: 77-91, 2015.

10. Ortiz ML, Lu L, Ramachandran I and Gabrilovich DI: Myeloid-derived suppressor cells in the development of lung cancer. Cancer Immunol Res 2: 50-58, 2014.

11. Dolcetti L, Peranzoni E, Ugel S, Marigo I, Fernandez Gomez A, Mesa C, Geilich M, Winkels G, Traggiai E, Casati A, et al: Hierarchy of immunosuppressive strength among myeloid-derived suppressor cell subsets is determined by GM-CSF. Eur J Immunol 40: 22-35, 2010.

12. Fujimura T, Mahnke K and Enk AH: Myeloid derived suppressor cells and their role in tolerance induction in cancer. J Dermatol Sci 59: 1-6, 2010.

13. Qu P, Wang LZ and Lin PC: Expansion and functions of myeloid-derived suppressor cells in the tumor microenvironment. Cancer Lett 380: 253-256, 2016

14. Schilling B, Sucker A, Griewank K, Zhao F, Weide B, Görgens A, Giebel B, Schadendorf D and Paschen A: Vemurafenib reverses immunosuppression by myeloid derived suppressor cells. Int J Cancer 133: 1653-1663, 2013.

15. Vetsika EK, Koinis F, Gioulbasani M, Aggouraki D, Koutoulaki A, Skalidaki E, Mavroudis D, Georgoulias V and Kotsakis A: A circulating subpopulation of monocytic myeloid-derived suppressor cells as an independent prognostic/predictive factor in untreated non-small lung cancer patients. J Immunol Res 2014 659294, 2014.

16. Schmid MC and Varner JA: Myeloid cells in the tumor microenvironment: Modulation of tumor angiogenesis and tumor inflammation. J Oncol 2010: 201026, 2010.

17. Solito S, Marigo I, Pinton L, Damuzzo V, Mandruzzato S and Bronte V: Myeloid-derived suppressor cell heterogeneity in human cancers. Ann N Y Acad Sci 1319: 47-65, 2014

18. Mandruzzato S, Solito S, Falisi E, Francescato S, Chiarion-Sileni V, Mocellin S, Zanon A, Rossi CR, Nitti D, Bronte $\mathrm{V}$ and Zanovello P: IL4Ralpha+ myeloid-derived suppressor cell expansion in cancer patients. J Immunol 182 6562-6568, 2009.

19. Mirsadraee S, Oswal D, Alizadeh Y, Caulo A and van Beek E Jr: The 7th lung cancer TNM classification and staging system: Review of the changes and implications. World J Radiol 4: 128-134, 2012.

20. Ridge CA, McErlean AM and Ginsberg MS: Epidemiology of lung cancer. Semin Intervent Radiol 30: 93-98, 2013.

21. Vinay DS, Ryan EP, Pawelec G, Talib WH, Stagg J, Elkord E, Lichtor T, Decker WK, Whelan RL, Kumara HM, et al: Immune evasion in cancer: Mechanistic basis and therapeutic strategies. Semin Cancer Biol 35 (Suppl): S185-S198, 2015.

22. Parcesepe P, Giordano G, Laudanna C, Febbraro A and Pancione M: Cancer-associated immune resistance and evasion of immune surveillance in colorectal cancer. Gastroenterol Res Pract 2016: 6261721, 2016.

23. Curiel TJ: Regulatory T cells and treatment of cancer. Curr Opin Immunol 20: 241-246, 2008
24. Stewart TJ and Abrams SI: How tumours escape mass destruction. Oncogene 27: 5894-5903, 2008.

25. Berzofsky JA and Terabe M: NKT cells in tumor immunity: Opposing subsets define a new immunoregulatory axis. J Immunol 180: 3627-3635, 2008

26. Marvel D and Gabrilovich DI: Myeloid-derived suppressor cells in the tumor microenvironment: Expect the unexpected. J Clin Invest 125: 3356-3364, 2015.

27. Adams JL, Smothers J, Srinivasan R and Hoos A: Big opportunities for small molecules in immuno-oncology. Nat Rev Drug Discov 14: 603-622, 2015

28. Mahoney KM, Rennert PD and Freeman GJ: Combination cancer immunotherapy and new immunomodulatory targets. Nat Rev Drug Discov 14: 561-584, 2015.

29. Sinha P and Ostrand-Rosenberg S: Myeloid-derived suppressor cell function is reduced by Withaferin A, a potent and abundant component of Withania somnifera root extract. Cancer Immunol Immunother 62: 1663-1673,2013.

30. Vincent J, Mignot G, Chalmin F, Ladoire S, Bruchard M, Chevriaux A, Martin F, Apetoh L, Rébé C and Ghiringhelli F: 5-Fluorouracil selectively kills tumor-associated myeloid-derived suppressor cells resulting in enhanced T cell-dependent antitumor immunity. Cancer Res 70: 3052-3061, 2010.

31. Yang WC, Ma G, Chen SH and Pan PY: Polarization and reprogramming of myeloid-derived suppressor cells. J Mol Cell Biol 5: 207-209, 2013.

32. Feng PH, Lee KY, Chang YL, Chan YF, Kuo LW, Lin TY, Chung FT, Kuo CS, Yu CT, Lin SM, et al: CD14(+)S100A9(+) monocytic myeloid-derived suppressor cells and their clinical relevance in non-small cell lung cancer. Am J Respir Crit Care Med 186: 1025-1036, 2012.

33. De Keersmaecker B, Fostier K, Corthals J, Wilgenhof S, Heirman C, Aerts JL, Thielemans K and Schots R: Immunomodulatory drugs improve the immune environment for dendritic cell-based immunotherapy in multiple myeloma patients after autologous stem cell transplantation. Cancer Immunol Immunother 63: 1023-1036, 2014

34. Corzo CA, Condamine T, Lu L, Cotter MJ, Youn JI, Cheng P, Cho HI, Celis E, Quiceno DG, Padhya T, et al: HIF-1 $\alpha$ regulates function and differentiation of myeloid-derived suppressor cells in the tumor microenvironment. J Exp Med 207: 2439-2453, 2010.

35. Kumar V and Gabrilovich DI: Hypoxia-inducible factors in regulation of immune responses in tumour microenvironment. Immunology 143: 512-519, 2014

36. Krstic J and Santibanez JF: Transforming growth factor-beta and matrix metalloproteinases: Functional interactions in tumor stroma-infiltrating myeloid cells. Scientific World Journal 2014: 521754, 2014

37. Beury DW, Parker KH, Nyandjo M, Sinha P, Carter KA and Ostrand-Rosenberg S: Cross-talk among myeloid-derived suppressor cells, macrophages, and tumor cells impacts the inflammatory milieu of solid tumors. J Leukoc Biol 96: $1109-1118,2014$

38. O'Garra A, Barrat FJ, Castro AG, Vicari A and Hawrylowicz C: Strategies for use of IL-10 or its antagonists in human disease. Immunol Rev 223: 114-131, 2008.

39. Eruslanov E, Neuberger M, Daurkin I, Perrin GQ, Algood C, Dahm P, Rosser C, Vieweg J, Gilbert SM and Kusmartsev S: Circulating and tumor-infiltrating myeloid cell subsets in patients with bladder cancer. Int J Cancer 130: 1109-1119, 2012.

40. Xiu B, Lin Y, Grote DM, Ziesmer SC, Gustafson MP, Maas ML, Zhang Z, Dietz AB, Porrata LF, Novak AJ, et al: IL-10 induces the development of immunosuppressive CD14(+)HLA-DR (low/-) monocytes in B-cell non-Hodgkin lymphoma. Blood Cancer J 5: e328, 2015.

41. Wang R, Lu M, Zhang J, Chen S, Luo X, Qin Y and Chen H: Increased IL-10 mRNA expression in tumor-associated macrophage correlated with late stage of lung cancer. J Exp Clin Cancer Res 30: 62, 2011.

42. Lievense LA, Bezemer K, Aerts JG and Hegmans JP: Tumor-associated macrophages in thoracic malignancies. Lung Cancer 80: 256-262, 2013.

43. Zeni E, Mazzetti L, Miotto D, Lo Cascio N, Maestrelli P, Querzoli P, Pedriali M, De Rosa E, Fabbri LM, Mapp CE and Boschetto P: Macrophage expression of interleukin-10 is a prognostic factor in nonsmall cell lung cancer. Eur Respir J 30: 627-632, 2007.

44. Wesolowski R, Markowitz J and Carson WE III: Myeloid derived suppressor cells-a new therapeutic target in the treatment of cancer. J Immunother Cancer 1: 10, 2013. 
45. Quatromoni JG and Eruslanov E: Tumor-associated macrophages: Function, phenotype, and link to prognosis in human lung cancer. Am J Transl Res 4: 376-389, 2012.

46. Ngiow SF, Teng MW and Smyth MJ: A balance of interleukin-12 and -23 in cancer. Trends Immunol 34: 548-555, 2013.

47. Ostrand-Rosenberg S, Sinha P, Beury DW and Clements VK: Cross-talk between myeloid-derived suppressor cells (MDSC), macrophages and dendritic cells enhances tumor-induced immune suppression. Semin Cancer Biol 22: 275-281, 2012.
48. Baird AM, Leonard J, Naicker KM, Kilmartin L, O'Byrne KJ and Gray SG: IL-23 is pro-proliferative, epigenetically regulated and modulated by chemotherapy in non-small cell lung cancer. Lung Cancer 79: 83-90, 2013. 